\title{
MOBILIDADE DE CONTEÚDOS EDUCACIONAIS: ADICIONANDO RECURSOS COMPLEMENTARES E INTERAÇÃO AO PAPEL
}

\author{
Núbia dos Santos Rosa Santana dos Santos, PGIE/UFRGS,nubia@pgie.ufrgs.br \\ José Valdeni de Lima, PGIE/UFRGS, valdeni@inf.ufrgs.br \\ Leandro Krug Wives, Instituto de Informática/UFRGS, wives@inf.ufrgs.br
}

\begin{abstract}
Resumo. $O$ acesso a conteúdos educacionais pode ser realizado usando diferentes tecnologias tais como, o computador, a Internet, os dispositivos móveis, TV interativa, etc., cada qual com diferentes restrições $e$, principalmente, potencialidades. Diante dessas possibilidades de acesso ao conteúdo educacional, deve-se analisar alternativas para tirar melhor proveito das potencialidades de cada uma, visando maior integração, mobilidade e interatividade do conteúdo. Este artigo apresenta uma proposta para acesso ao conteúdo educacional e um experimento com resultados que apresentam a relevância da construção de conteúdos educacionais com mobilidade, interatividade e uso adequado de recursos multimídia.
\end{abstract}

Palavras-chaves: mobilidade, interatividade, mídia impressa

\section{MOBILITY OF EDUCATIONAL CONTENT: ADDING RESOURCES AND INTERACTION TO THE PAPER}

\begin{abstract}
The access to educational content can be realized using different technologies such as computer, Internet, mobile devices, interactive TV, etc., each with different restrictions, and especially, potential. In face these possibilities of access to educational content, we should analyze alternatives to advantage of the best capabilities of each one, seeking greater integration, mobility and interactivity of content. This paper presents a proposal for access to educational content and an experiment with results that show the importance of building educational content with mobility, interactivity and appropriate use of multimedia resources.
\end{abstract}

Keywords: mobility, interactivity, printed media

\section{1- Introdução}

Diversos dispositivos e tecnologias estão à disposição de alunos e professores: o computador, a internet, os dispositivos móveis (incluindo celulares, smartphones, tablets) e até mesmo a TV digital. $\mathrm{O}$ acesso a conteúdos pedagógicos pode ser feito usando essas diferentes tecnologias. Diante de tantas possibilidades, é fundamental elaborar estratégias que possibilitem a continuidade do ensino através de diferentes dispositivos, possibilitando ao aluno acessar a informação independente da tecnologia e do local. 
Vale ressaltar que tais conteúdos educacionais, como os Objetos de Aprendizagem (OA), podem ser utilizados no ensino presencial e também na modalidade a distância. Mas, segundo dados do Censo Ead.Br (2010), a mídia mais utilizada no ensino a distância ainda é a impressa (87,2\% das instituições), seguida por e-learning (71.5\%) e vídeo (51.7\%). De acordo com o ENAP (2006), o material impresso é um complemento importante de outros materiais (e.g., vídeos e programas de TV), e, mesmo no caso de cursos pela Internet, a observação tem mostrado que os alunos tendem a imprimir qualquer texto que ultrapasse quatro ou cinco páginas.

Nesse contexto onde os alunos efetivamente costumam imprimir o material educacional, torna-se relevante verificar alternativas para integrar os benefícios do material impresso com a interatividade presente nos recursos digitais. Dessa forma, deve-se pensar em estratégias que integrem a mobilidade do papel e a possibilidade dos recursos interativos presentes no e-learning de forma a favorecer o ensino das pessoas que possuem dificuldade em acesso ao conteúdo educacional via e-learning ou mesmo para alunos de cursos na modalidade presencial que preferem imprimir o conteúdo para estudar.

Este artigo apresenta a integração de códigos 2D (também conhecidos por etiquetas ou tags visuais) na mídia impressa, possibilitando ao aluno interagir de forma semelhante ao que é feito no e-learning, desde que utilize um dispositivo móvel ( $\mathrm{m}$ learning). Segundo Patokorpi et al. (2007) m-learning possibilita aprendizagem como ensino colaborativo e orientado, suportados por dispositivos móveis que utilizam canais de comunicações móveis, e que dentre outras possibilidades pode servir como apoio ao ensino tradicional.

O documento está organizado da seguinte forma. A seção seguinte apresenta conceitos relacionados à mobilidade e interatividade. A seção 3 apresenta a proposta $\mathrm{e}$ a seção 4 apresenta um experimento realizado usando a mídia impressa e os recursos do e-learning. A seção 5 apresenta considerações finais sobre o estudo realizado.

\section{2- $\quad$ Mobilidade e Interatividade}

A mobilidade é uma das características da mídia impressa. Outras características dela são apresentadas por Sellen e Harper (2001), incluindo: a possibilidade de navegação rápida e flexível através de um documento, a inserção de anotações durante a leitura, e a integração de atividades híbridas, tais como escrita e leitura.

No entanto, Belisário (2006) apresenta algumas limitações em relação ao uso do material impresso, o qual se caracteriza pela pouca interatividade, servindo mais para a leitura do que para estudo. O papel também não possui áudio nem vídeo. Segundo Moore e Kearsley (2008), o vídeo e o áudio são especialmente eficazes para a transmissão de aspectos emocionais ou relacionados à atitude de uma disciplina, podendo ser usados para apresentar opiniões de especialistas, aumentando a credibilidade e o interesse pelos materiais.

Tais limitações podem ser reduzidas com o uso de dispositivos móveis, que possuem características interessantes. Patokorpi et al. (2007), por exemplo, destacam alguns recursos relevantes da tecnologia móvel:

- Mobilidade. Artefatos tecnológicos podem ser usados enquanto estiverem em movimento; 
- $\quad$ Ubiquidade. A tecnologia não é só portátil e móvel, mas também uma parte integrante do ambiente do usuário;

- Multimodalidade. Interação da tecnologia pelo usuário pode ter lugar e muitas modalidades, dando ao usuário a liberdade de interagir com a tecnologia de uma maneira que seja conveniente;

- Interatividade. O usuário e o sistema tecnológico podem interagir um com o outro e essa interação não é apenas controlada pelo usuário, pois também pode ser iniciada pela tecnologia;

- $\quad$ Personalização. O usuário ou o sistema pode personalizar as definições operacionais de acordo com as preferências do usuário.

Observa-se que o uso de dispositivos móveis possibilita a mobilidade e interatividade ao mesmo tempo, mas exige com que o dispositivo esteja ligado e, eventualmente, conectado. Diante disso, por que não unir as vantagens oferecidas por ambos?

Pelo fato de o papel estar sempre disponível (i.e., não necessita estar "ligado"), ele oferece maior mobilidade ao material educacional. Se pudermos agregar ao papel alguma forma ou mecanismo capaz de estabelecer referências com outras mídias ou materiais, podemos agregar interatividade a ele (e poderia agir ainda como mecanismo de entrada de dados). Dessa forma, alunos poderiam acessar recursos dinâmicos (e.g., vídeos ou animações) através do papel. Falta, então, identificar maneiras para estabelecer tais referências e tornar o papel um mecanismo de entrada de dados efetivo.

Algumas possibilidades de tecnologias apropriadas para reconhecer o papel como dispositivo de entrada de dados de forma computacional são apresentadas em Klemmer (2003):

-Etiquetas eletrônicas passivas (e.g., etiquetas RFID - Radio Frequency IDentification e IR - Infra-Red);

-Etiquetas eletrônicas ativas (e.g., motes);

-Etiquetas visuais (inclui 2D, glyphs e QR-Codes);

-Análise de conteúdo baseada no processamento de imagens (e.g., OCR Optical Character Recognition).

Entre tais recursos, destacam-se os códigos 2D, que são figuras de duas dimensões que armazenam informações que podem ser lidas por dispositivos eletrônicos compatíveis. Exemplos práticos de tecnologias que geram tais códigos são o DataMatrix e o QR Code ${ }^{1}$.

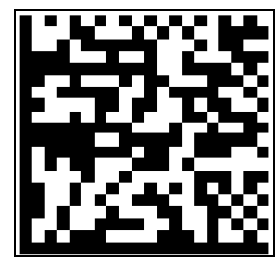

Figura 1. Exemplo de código DataMatrix

Fonte: Falas e Kashani (2007)

${ }^{1}$ QR Code é marca registrada da DENSO WAVE INCORPORATED. 
Segundo Falas e Kashani (2007), o DataMatrix (Figura 1) possui maior capacidade de dados. O QR-Code é um código de barras de duas dimensões criado pela empresa japonesa Denso Wave (2010), que pode ser lido em dispositivos móveis (celular com câmera) ou computadores (e.g., laptops).

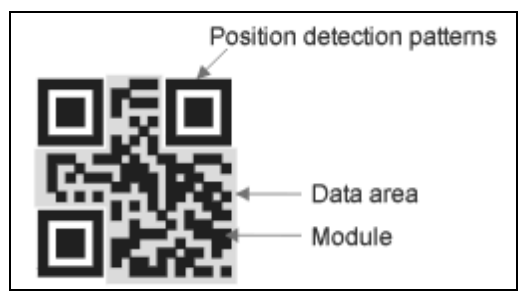

Figura 2. Exemplo de QR Code

Fonte: http://www.denso-wave.com/qrcode/qrfeature-e.html

Quando acessado, o QR Code permite realizar ações como acessar um site, ler um texto, informar dados para envio de mensagens (SMS) e contatos. O QR Code possui maior velocidade de processamento e capacidade de dados do que o DataMatrix (Denso-Wave, 2010).

\section{3- $\quad$ Proposta}

A proposta é utilizar a mídia impressa (integrada a tecnologia QR Code) como um meio alternativo para fornecer a aprendizagem com mobilidade e como mecanismo de interação e/ou entrada para outros dispositivos buscando a interatividade. O QR Code foi utilizado devido as suas características de processamento e capacidade de dados.

Usando a tecnologia QR Code na mídia impressa todos os recursos presentes no material, tais como textos, imagens, áudios, vídeos, etc. poderão ser acessados de forma integrada e/ou individualmente, usando um dispositivo móvel (Figura 3).

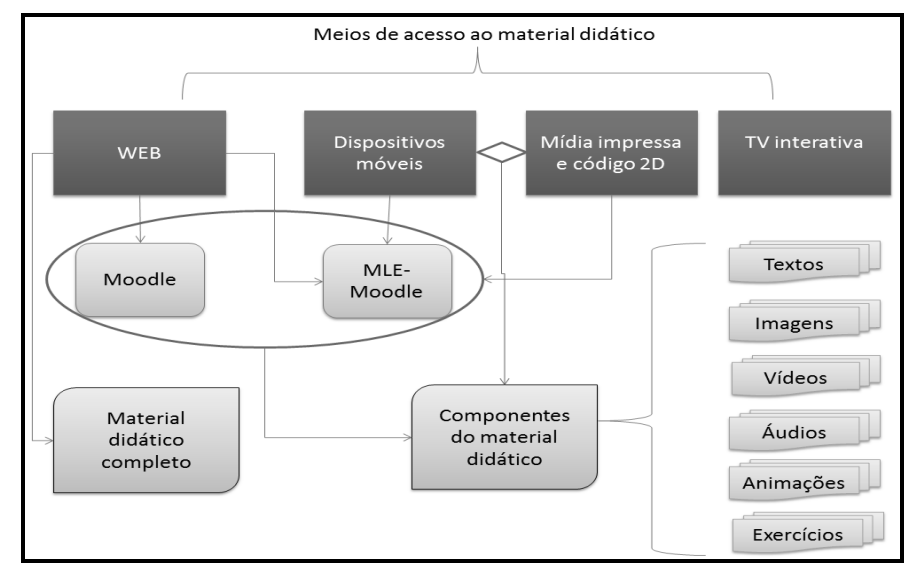

Figura 3. Meios de acesso ao material (completo ou por componentes)

Dessa forma, o material permite acesso a elementos externos, estáticos (como textos e imagens) e também a elementos dinâmicos, como vídeos, animações, etc., os quais podem ser acessados de forma integrada ao contexto ou separadamente. $\mathrm{O}$ aluno pode visualizar o conteúdo textual e imagens e ao mesmo tempo ter a possibilidade de 
acessar todo o conteúdo multimídia do material, através dos links disponíveis nos códigos 2D.

Nesta proposta, os códigos 2D são específicos de acordo com o tipo de interação, por exemplo, para acessar um vídeo o código possui um ícone específico e diferente de um código para acesso a um áudio ou a um exercício. Essa estratégia busca facilitar a orientação e a organização do conteúdo. Para a visualização de uma animação, vídeo, áudio, ou conteúdo extra (e.g., página Web) referente ao conteúdo, o aluno pode utilizar o QR-Code. O aluno com um celular com câmera e acesso a Internet conseguirá acessar qualquer recurso dinâmico relacionado ao conteúdo educacional.

Um OA foi desenvolvido seguindo a proposta apresentada. O OA é composto de pequenos componentes digitais, os quais podem ser descritos por metadados. O conteúdo do OA desenvolvido aborda a temática "Prevenção no uso da Internet" e tem como referencial as dicas fornecidas pela Cartilha de Segurança do CGI e SaferNet e, ainda, baseado em um material desenvolvido pelo curso de Informática Instrumental ${ }^{2}$.

O OA foi elaborado em HTML5 e Javascript, tendo sido disponibilizado no ambiente Moodle 3 . Conforme apresenta a Figura 3 pode-se usar o MLE-Moodle, um plugin do Moodle 4 . O OA é composto de diversos recursos multimídias (vídeo, áudio, animação), e foi elaborado seguindo princípios da aprendizagem multimídia (Mayer, 2009), tais como:

- Princípio da multimídia - as pessoas aprendem melhor a partir de palavras e imagens do que apenas palavras. Imagens foram utilizadas como complemento ao texto;

- Princípio da proximidade espacial - os alunos aprendem melhor quando as palavras e as imagens correspondentes são apresentadas perto do que longe de cada uma na página ou na tela. Para Filatro (2008), quando as pessoas precisam integrar informação verbal e pictórica (e.g. gráfico e explicação na tela de como ler o gráfico) pode ocorrer uma sobrecarga na memória de trabalho e o processo de aprendizado ser perturbado. No OA os elementos de informação de um determinado contexto foram integrados na mesma página;

- Princípio da Contiguidade Temporal - alunos aprendem melhor quando as palavras e imagens correspondentes são apresentadas simultaneamente em vez de sucessivamente. As imagens e palavras do OA aparecem simultaneamente;

- Princípio da coerência - as pessoas aprendem mais profundamente a partir de uma mensagem multimidia, quando um material não relacionado (não relevante) está excluído. Algumas imagens foram analisadas e retiradas devido a não relevância ao contexto;

- Princípio da sinalização - As pessoas aprendem melhor a partir de uma mensagem multimídia, quando o texto é sinalizado em vez de não sinalizado. Algumas partes do texto foram sinalizadas através do uso de negrito;

- Personalização - as pessoas aprendem melhor a partir de apresentações multimídia quando as palavras estão em estilo coloquial, em vez de estilo formal.

\footnotetext{
2 Curso de Informática Instrumental para Professores da Educação Básica - UAB UFRGS, Material elaborado pela Professora Taisy Weber - UFRGS

3 Moodle (Modular Object Oriented Developmental Learning Environment) é um Sistema Open Source de Gerenciamento de Cursos, um Ambiente Virtual de Aprendizagem (AVA). Disponível em: http://moodle.org/about/

4 MLE-Moodle permite a exploração do conteúdo através de dispositivos móveis. Disponível http://mle.sourceforge.net/
} 
O OA é formado por textos, áudio referente aos textos (caso o aluno prefira ouvir o conteúdo), vídeos, animações e links para conteúdos extras, fórum de discussão, wiki e questionários.

\section{4- Experimento}

Esta seção apresenta um experimento realizado com o objetivo de analisar o desempenho de alunos usando meios diferentes de estudo (material digital e material impresso) e ainda verificar como os alunos costumam estudar o material disponibilizado pelo professor. A proposta objetiva a elaboração de conteúdo para o ensino a distância, assim como para o ensino presencial. $O$ experimento foi realizado em sala, presencialmente, para que as condições fossem as mesmas a todos os alunos, ter um controle e evitar resultados equivocados.

O experimento foi realizado com uma turma da disciplina de Computação e Sistemas de Informação. De acordo com a metodologia utilizada a turma foi dividida em três grupos. O primeiro grupo de alunos usando o computador com acesso a Internet, o segundo grupo de alunos usando somente o papel e o terceiro com alunos usando o papel (com QR Code) com dispositivos móveis.

No entanto, não foi possível compor o terceiro grupo devido a falta de dispositivos com configuração adequada para instalação do leitor de QR Code. Dessa forma, a estratégia foi aplicada com a turma dividida em dois grupos:

- Grupo A - alunos no computador com Internet

- Grupo B - alunos usando o papel

A mídia impressa (Figura 4) contém o mesmo conteúdo da Web e os recursos como vídeo, áudio, links foram representados por QR Code e poderiam ser explorados com o uso de um dispositivo móvel, tais como celulares.

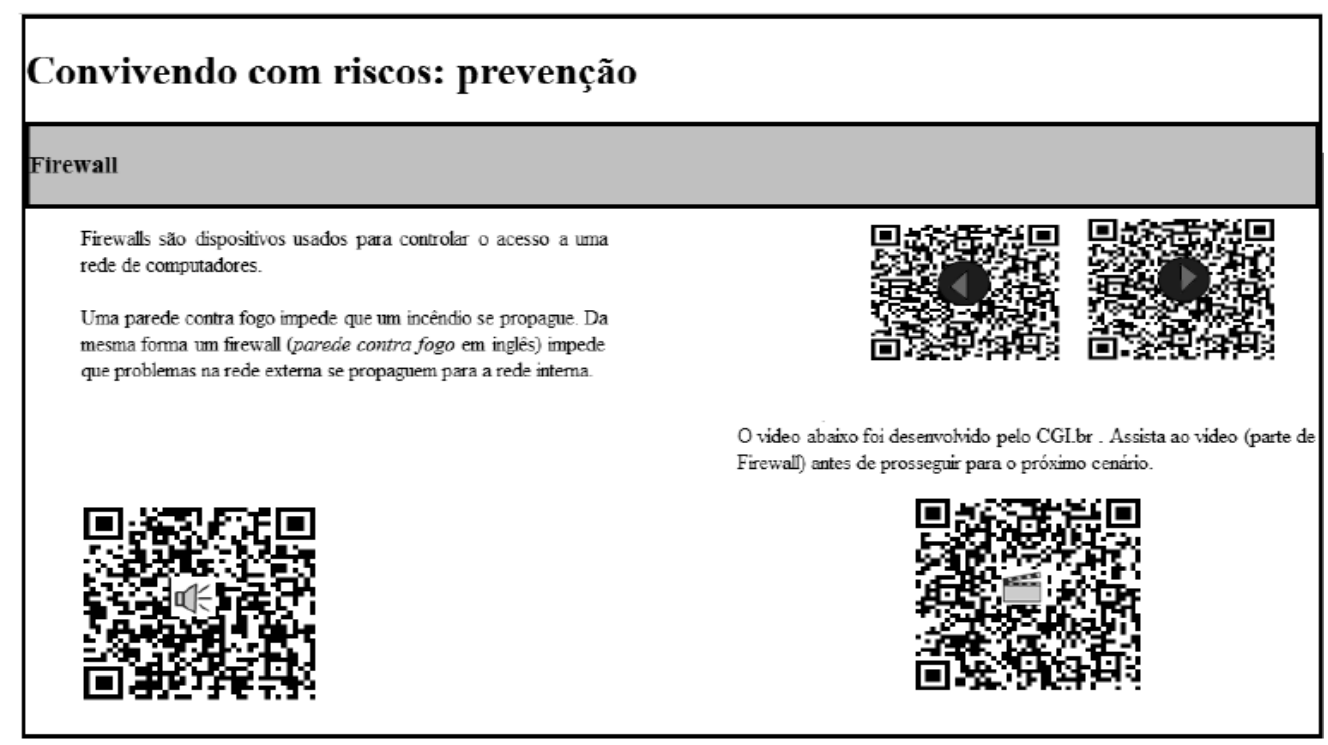

Figura 4. Tela do OA impresso com QR Code

Todos os recursos existentes no OA acessado na Web foram materializados no papel. Observa-se que os recursos representados por QR Code possuem ícones (Figura 
4) para representar a funcionalidade como, por exemplo: acessar um áudio, acessar um vídeo, ir para a próxima página, retornar a página anterior, etc. A Figura 5 apresenta o mesmo OA acessado pela Internet.

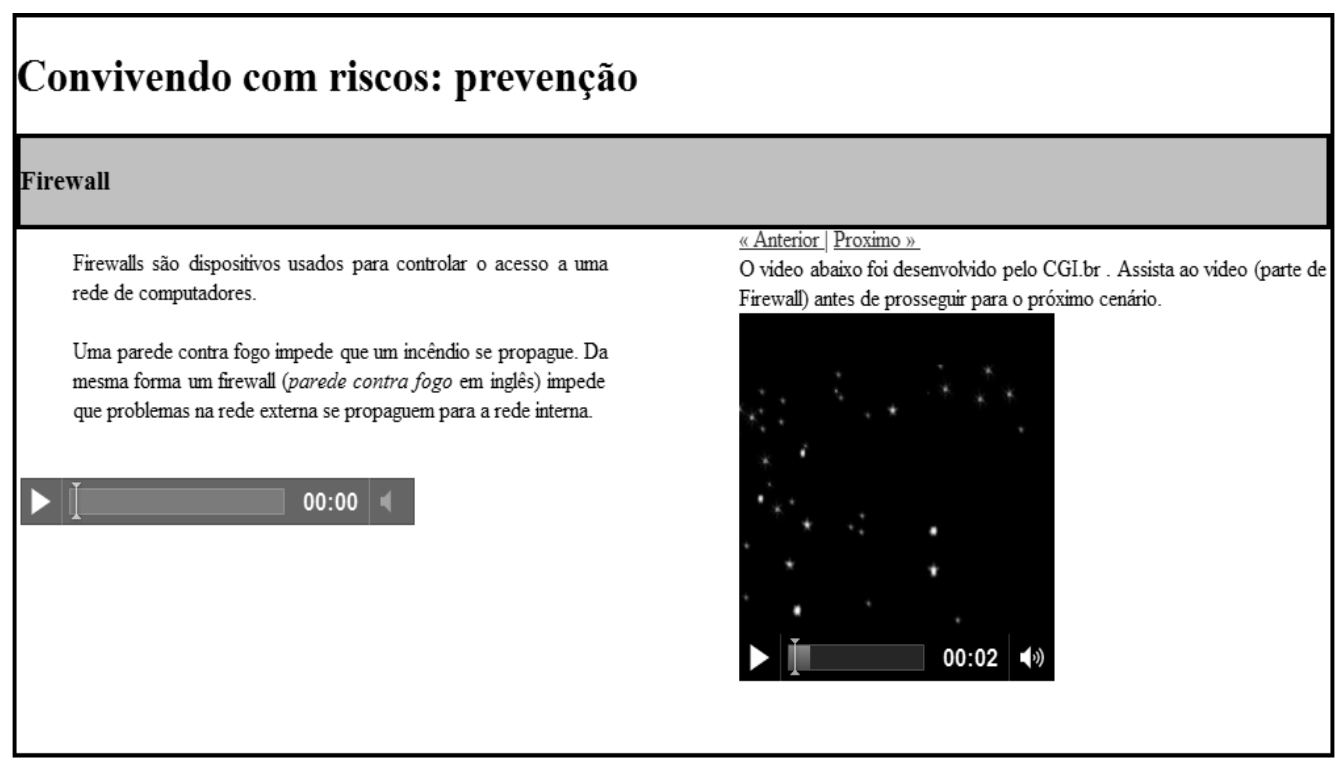

Figura 5. Tela do OA na Web (no Moodle)

No final do conteúdo do OA foram inseridas algumas questões. As questões foram elaboradas considerando apenas as informações comuns no papel e na Web, não considerando a necessidade de acesso a vídeos, áudios, animações, etc., justamente para que o resultado não fosse tendencioso.

No experimento, em um total de 10 alunos, o Grupo A foi formado por 5 alunos e o Grupo B por 5 alunos.

A Figura 6 apresenta os resultados da análise das respostas dos alunos referentes às questões.

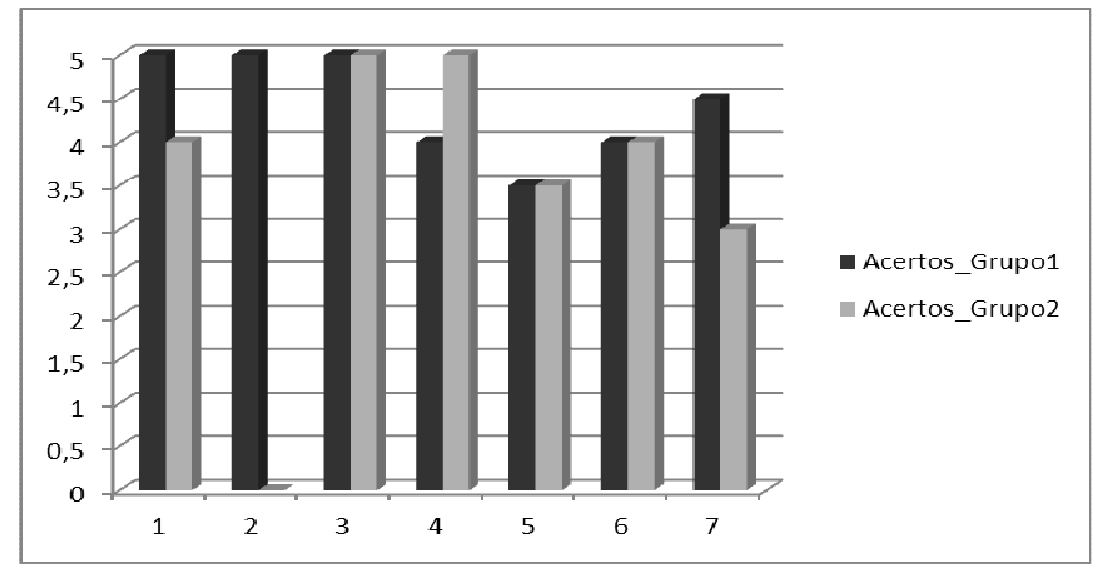

Figura 6. Número de acertos do Grupo 1 e Grupo 2

O questionário foi composto de 7 questões, sendo 4 objetivas e 3 discursivas. Conforme mostra o gráfico da Figura 6 na questão 1, questão 2 e questão 7 o Grupo A 
obteve mais acertos. Enquanto na questão 4 o Grupo B obteve mais acertos. Nas questões 3,5 e 6 o número de acertos foi o mesmo.

Considerando a média dos acertos de cada grupo verifica-se que o Grupo A obteve mais acertos do que o Grupo B (Figura 7).

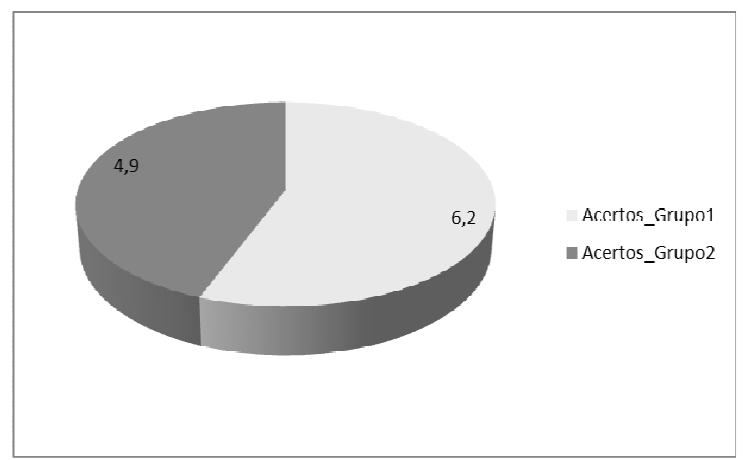

Figura 7. Comparativo entre médias de acertos dos dois Grupos

A média de acertos do Grupo A foi de 6,2 enquanto a média de acertos do Grupo B foi de 4,9. Vale ressaltar que de acordo com as informações fornecidas pelos alunos o Grupo B possui mais conhecimento prévio sobre o conteúdo do que o Grupo A (Figura 8) o que deveria neste caso, apresentar um resultado contrário ao que foi apresentado.

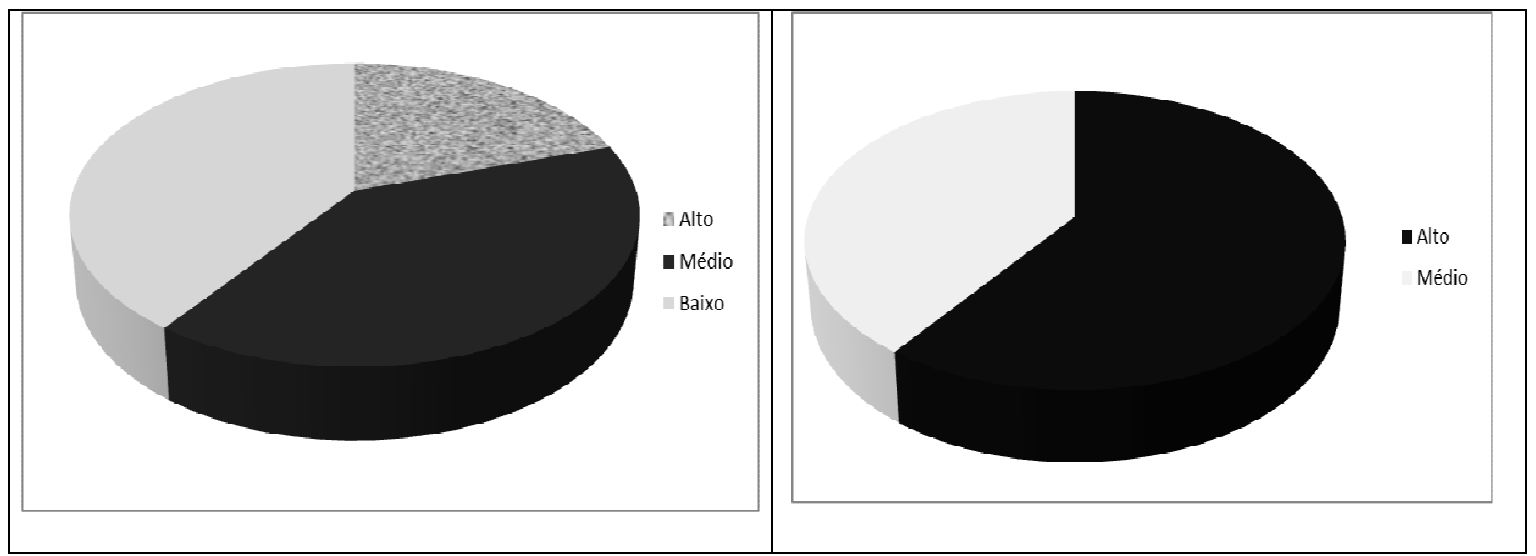

Figura 8. Conhecimento prévio Grupo A e Conhecimento prévio Grupo B, respectivamente

Dos cinco alunos que responderam ao questionário sobre o uso dos recursos multimídia no OA, todos relataram que os recursos existentes no OA auxiliaram no entendimento do conteúdo. Segundo o relato de um aluno sobre os recursos multimídia presentes no OA: "os recursos multimídia auxiliaram, são didáticos. E os links igualmente, com ótimas recomendações de sites". Já um dos alunos apontou que entre os recursos existentes a animação não auxiliou.

Os alunos do Grupo B responderam ao mesmo questionário, porém não puderam responder a essas questões uma vez que utilizaram somente o papel e por isso não puderam explorar os recursos dinâmicos, como animações, vídeos e áudio.

Do Grupo A três alunos informaram que aprendem mais ouvindo do que lendo o conteúdo, neste caso, utilizam mais o canal verbal. Daí a importância de se elaborar conteúdos que favorecem os diferentes estilos cognitivos.

Conforme apresenta o Quadro 1, os alunos quando questionados sobre o meio para acesso e estudo do material, a maioria afirmou que de acordo com a situação, costumam imprimir o material para estudar. 


\begin{tabular}{|l|c|}
\hline \multicolumn{1}{|c|}{ Situações } & Quantidade de alunos \\
\hline Imprime sempre & 1 \\
\hline Imprime a partir de 3 páginas & 1 \\
\hline Imprime a partir de 4 páginas & 1 \\
\hline $\begin{array}{l}\text { Imprimem a partir de 10 } \\
\text { páginas }\end{array}$ & 2 \\
\hline $\begin{array}{l}\text { Imprime se usar o conteúdo } \\
\text { várias vezes }\end{array}$ & 1 \\
\hline $\begin{array}{l}\text { Imprime dependendo da } \\
\text { complexidade do conteúdo }\end{array}$ & $\mathbf{2}$ \\
\hline Não imprimem & $\mathbf{2}$ \\
\hline $\begin{array}{l}\text { Imprimem somente o } \\
\text { essencial }\end{array}$ & 1 \\
\hline
\end{tabular}

Quadro 1. Uso do meio impresso

O relato de um aluno justifica o motivo de imprimir o conteúdo: "Gosto mais de estudar através do papel, onde posso fazer anotações, mas muitas vezes não imprimo, se puder usar o micro ou o note. Não depende do número de páginas para eu imprimir, depende da matéria e se o assunto é muito complexo". Esse relato pode indicar o papel como uma possibilidade de entender um conteúdo mais complexo, uma vez que possibilita fácil anotação e observação sobre o conteúdo estudado.

Outro aluno relata que: "Costumo imprimir acima de 4 (quatro) páginas, pois a iluminação da tela do computador acaba cansando a visão."

Considerando as situações em que os alunos costumam imprimir o material a ser estudado, o artigo propõe o uso da mídia impressa com códigos 2D como forma de integrar recursos multimídia que podem complementar o ensino. Por exemplo, se o aluno prefere imprimir o conteúdo porque este é mais complexo e permite fácil anotação, este conteúdo impresso pode conter códigos 2D que permitam acessar uma animação, vídeo ou conteúdo extra sobre esse assunto, colaborando para o entendimento de um conteúdo complexo. Considerando a relevância da integração de tecnologias, Özdemir (2010) ressalta que além da aprendizagem significativa, a integração de tecnologias móveis em livros impressos com a ajuda de códigos de barra 2D tem o potencial para reduzir a carga cognitiva, inclusive em ambientes de educação a distância.

Em relação a mobilidade destaca-se a possibilidade de o aluno dar continuidade aos estudos independente do local, aproveitando muitas vezes o seu tempo vago. Um exemplo disso é o relato de um aluno: "[]...muitas vezes estudo no caminho dos meus afazeres cotidianos, como ônibus, carro ou restaurante e lanchonetes onde faço minhas refeições usando folhas impressas ou escritas e as vezes notebook". Percebe-se com isso, a importância de elaborar materiais com mobilidade e que permitam a integração de tecnologias. Por exemplo, pode-se elaborar um OA para ser acessado na Internet (desktop, notebook), pela mídia impressa (com QR Code) e/ou por dispositivos móveis (celular, smartphones). $\mathrm{Na}$ ausência de um desses meios o aluno poderá dar continuidade aos estudos (mesmo OA) usando os outros meios alternativos.

\section{5- Considerações finais}

O experimento realizado não teve como objetivo destacar um meio ou outro para acesso ao conteúdo educacional, mas sim a integração de diferentes meios, uma vez que os 
usuários (alunos) costumam utilizar diferentes meios em diferentes situações. Diante do resultado do experimento, a hipótese é que o resultado em relação ao desempenho dos grupos pode ter sofrido alguma influência em relação aos meios complementares como os vídeos, áudios e animações. E por isso, o foco é apresentar possibilidades que complementem o material impresso através do uso de códigos 2D e dispositivos móveis para acesso a vídeos, áudio, animações e etc., e que favoreçam a aprendizagem com maior mobilidade.

O experimento realizado com os alunos possibilitou verificar que a integração de recursos complementares como vídeos, animações, informações extras são importantes para o entendimento do conteúdo.

Além disso, verificou-se que alguns alunos preferem imprimir o material para estudar devido às características de mobilidade e de realizar anotação. Observou-se também que alguns alunos preferem ouvir a ler o conteúdo. O material impresso pode conter códigos que direcionem para um mesmo conteúdo com a opção de áudio.

Dessa forma, a proposta de integração das tecnologias de código 2D ao material impresso pode colaborar para o ensino a distância ou mesmo presencial, uma vez que permite a mobilidade (através do papel) e a interatividade (e mobilidade) do $\mathrm{m}$ learning. Essa proposta será aplicada com alunos usando a mídia impressa ( com QR Code) e dispositivos móveis com capacidade de decodificação. Pretende-se com isso verificar a viabilidade da proposta para elaboração de conteúdos para o ensino a distância e também para o presencial.

\section{Referências}

BELISARIO, Aluízio. O material didático na educação a distância e a constituição de propostas interativas, In: SILVA, M. (org), Educação Online, São Paulo: Loyola, pp. 137-148. 2006.

CENSO Ead.br, Censo ead.br / organização Associação Brasileira de Educação a Distância. São Paulo: Pearson Education do Brasil.2010.

DENSO WAVE Incorporated, Basic Info, Disponível em http://www.densowave.com/en/adcd/fundamental/index.html, Acesso em: 14 Out. 2010.

ENAP, Educação a distância em organizações públicas; mesa-redonda de pesquisa-ação. Brasília: ENAP. 200p. 2006

FALAS, Tasos; KASHANI, Hossein, Two-Dimensional Bar-code Decoding with Camera-Equipped Mobile Phones. Proceedings of the Fifth Annual IEEE International Conference on Pervasive Computing and Communications Workshops - PerComW'07. White Plains, NY.2007.

FILATRO, Andrea, Design instrucional na prática, São Paulo: Pearson Education do Brasil. 2008.

KLEMMER, Scott et al., Books with Voices: Paper Transcripts as a Tangible Interface to Oral Histories, CHI 2003: NEW HORIZONS, Ft. Lauderdale, Florida, USA. pp.89-96. 2003.

MAYER, Richard, Multimedia Learning. Cambridge: Cambridge University Press. 2 ed. 2009.

MOORE, Michael.; KEARSLEY, Greg, Educação a distância: uma visão integrada, São Paulo: Cengage Learning.2008.

ÖZDEMIR, Selçuk, Supporting printed books with multimedia: A new way to use mobile technology for learning, British Journal of Educational Technology, Vol. 41 No 6, pp. 135138.2010 .

PATOKORPI, Erkki et al, Learning objects to support constructivist learning, In: Harman, K \& Koohang, A. (Eds), Learning objects: applications, implications \& future directions, Santa Rosa, Califórnia: Informing Science Press. pp. 187-221,2007

SELLEN, Abigail; HARPER, Richard, The Myth of the Paperless Office. Cambridge, MA: MIT Press.2001. 